\title{
Risk factors for ischemic stroke: differences between cerebral small vessel and large artery atherosclerosis aetiologies
}

\author{
Yan Shi ${ }^{1,2}$, Li Guo ${ }^{2}$, Yangkun Chen ${ }^{3}$, Qingfan $\mathrm{Xie}^{1}$, Zhenyu Yan ${ }^{1}$, Yongtao Liu ${ }^{1}$, Juxian Kang ${ }^{4}$, Shuang $\mathrm{Li}^{4}$ \\ ${ }^{1}$ Department of Rehabilitation Medicine, Xingtai People's Hospital, Xingtai, Hebei Province, China, ${ }^{2}$ Department of Neurology, \\ The Second Hospital of HeBei Medical University, Shijiazhuang, Hebei Province, China, ${ }^{3}$ Department of Neurology, Dongguan \\ People's Hospital (Affiliated Dongguan Hospital, Southern Medical University), Dongguan, Guangdong Province, China, ${ }^{4}$ Department \\ of Neurology, Xingtai People's Hospital, Xingtai, Hebei Province, China
}

\begin{abstract}
The treatment for large artery atherosclerosis (LAA) stroke is also important for patients with cerebral small vessel disease (SVD). Our aim is to clarify the risk factors for ischemic cerebral SVD and to evaluate the different risk factor profiles of the two ischemic stroke subtypes, namely, ischemic cerebral SVD and LAA. A total of 353 patients with acute ischemic stroke were assigned to two groups according to the Trial of ORG 10172 in Acute Stroke Treatment (TOAST) criteria: the ischemic cerebral SVD group and the LAA group. A total of 70 non-stroke patients admitted during the same period served as the control group. Clinical variables were collected, including age, sex, blood pressure, blood glucose, triglycerides, low-density lipoprotein, total cholesterol (TC), smoking history, drinking history, coronary atherosclerotic heart disease and family history of a high-risk disease. Multivariate logistic regression (MLR) analyses were performed. MLR analysis showed that risk factors for LAA stroke included hypertension, diabetes mellitus, high low-density lipoprotein, hypertriglyceridemia and smoking compared with the control group. The analysis also showed that the risk factors for cerebral SVD stroke were hypertension, diabetes mellitus, high TC, hypertriglyceridemia and smoking. In terms of the factors distinguishing LAA and cerebral SVD, MLR demonstrated that high TC played prominent roles in cerebral SVD. Hypertension, diabetes mellitus, high total cholesterol, hypertriglyceridemia and smoking are independent risk factors for cerebral SVD stroke. Compared with the LAA stroke group, patients with cerebral SVD stroke were more likely to have a high level of TC.
\end{abstract}

Key words: cerebral small vessel disease, white matter lesions, large artery atherosclerosis.

\section{Introduction}

Cerebral small vessel disease (SVD) refers to the syndrome of clinical, cognitive, imaging and pathological manifestations induced by lesions of the small cerebral perforating arteries and arterioles (diameter, 40-200 $\mu \mathrm{m}$ ), capillaries, and venules [32]. It is customary to refer to the clinical and imaging findings induced by lesions of the small perforating arteries and arterioles and the resulting brain damage in the cerebral white and deep grey matter [25]. These perforating vessels are essential to maintain optimum functioning of the brain's most metabolically active nuclei and complex white matter networks [3]. The imaging features of ischemic SVD are lacunes, white 
matter hyperintensity (WMH), cerebral microbleeds (CMB), enlarged perivascular spaces (EPVS) and brain atrophy (BA) [31].

Cerebral SVD accounts for approximately onequarter of all ischemic strokes, which is more than twice the risk of recurrent stroke [23]. Studies have shown that SVD is more common in the Chinese than in the Westerners [34]. Cerebral SVD is not only a leading cause of lacunar stroke, cognitive dysfunction, dizziness, unstable gait and urination disorders, but it can also lead to senile depression, Parkinsonism, and accounts for up to $45 \%$ of dementias [27] The most common types of cerebral SVD are age-related cerebral SVD and hypertension-related cerebral SVD, which are considered as the risk factors for ischemic stroke [2].

Large artery atherosclerosis (LAA) is one of the most common subtypes of ischemic stroke [9], which is recognized as the leading reason for cerebrovascular diseases. In terms of risk factors and pathogenesis, it is reported that there is a positive correlation between LAA stroke and cerebral SVD [22]. In the recent clinical application, it has been observed that the different MRI features of cerebral SVD in the patients who are undergoing ischemic stroke caused by LAA [26]. As we know, treatment for LAA stroke is also important for patients with cerebral SVD.

The presence of cerebral SVD has a major impact on some conventional therapies. Therefore, early detection and treatment of vascular risk factors for cerebral SVD are of great significance. This study aimed to determine the risk factors for arteriolosclerosis-induced ischemic cerebral SVD and to compare them with those of LAA.

\section{Material and methods \\ Study subjects}

This was a retrospective observational cohort study. A total of 353 patients admitted to our hospital with first-ever acute ischemic stroke confirmed by cranial magnetic resonance imaging (MRI) were consecutively enrolled between January 2011 and December 2012.

The inclusion criteria were: 1) acute ischemic first-ever stroke within 7 days of admission; 2) age between 35 and 90 years old; 3) classified as LAA or cerebral SVD (lacunar infarction or lacunar infarction with evidence of white matter lesions). The aetiology of acute ischemic stroke was classified in accor- dance with the Trial of ORG 10172 in Acute Stroke Treatment (TOAST) classification. The TOAST classification system is a widely used method for classifying ischemic stroke and involves the division of strokes into different subtypes according to clinical and neuroimaging information [19].

The exclusion criteria were: 1) cardioembolism, stroke of other undetermined aetiology, acute stroke of other determined aetiology; 2) potential cardiogenic emboli, infections, malignant diseases, severe heart disease, heart, liver, or kidney failure, poisoning, specific white matter diseases, multiple sclerosis, hypercoagulability, hydrocephalus, acute disseminated encephalomyelitis, hypoxia, or adrenal white matter malnutrition; 3) stroke history, mixed strokes (haemorrhage and then infarction or infarction and then haemorrhage), arteritis-induced cerebral infarctions, malignant tumours, alimentary tract haemorrhage, subarachnoid haemorrhage, cerebral haemorrhage, or pregnancy and lactation; 4) patients who had insufficient examinations after admission in whom an etiological analysis.

\section{Collection of clinical data}

All of the included patients completed a unified case registration form. We comprehensively collected patients' clinical features, including their vital signs, past medical history, personal history, family history, and clinical signs. After admission, electrocardiography (ECG), echocardiography, and cranial MRI, computed tomography angiography (CTA) or magnetic resonance angiography (MRA) of the head and neck were performed. A volume of $10 \mathrm{ml}$ of fasting venous blood from the cubital vein was collected in a vacuum tube between 6 and 7 o'clock in the morning after admission (after more than 8 hours on an empty stomach). Blood glucose and blood lipid levels (TC, low-density lipoprotein [LDL], and triglyceride [TG] levels) were measured. The study was approved by the Ethics Committee of Xingtai People's Hospital. Informed consent was obtained from all patients.

\section{Unified standards of risk factors}

Hypertension [15]: Hypertension was defined as a systolic blood pressure (SBP) $\geq 140 \mathrm{mmHg}$ and/or a diastolic blood pressure (DBP) $\geq 90 \mathrm{mmHg}$ after blood pressure was measured three times on different days or if patients had previously identified hypertension and were taking antihypertensive drugs. 
Diabetes mellitus [5]: Diabetes mellitus was defined in accordance with the Chinese Diabetes Prevention Guide Standards, 2010 version. Patients were considered to have diabetes if their blood glucose was $\geq 11.1 \mathrm{mmol} / \mathrm{l}$, if their fasting blood glucose was $\geq 7.0 \mathrm{mmol} / \mathrm{l}$ at least twice, or if they had a previous history of diabetes mellitus and were taking hypoglycaemic drugs, except for patients with type 1 diabetes mellitus.

Coronary heart disease: Coronary heart disease (CAD) was considered as a kind of heart disease caused by coronary artery stenosis or obstruction caused by atherosclerotic lesions, resulting in myocardial ischemia, hypoxia or necrosis. Coronary atherosclerotic heart disease was verified by coronary angiography or coronary CTA or by consultation with cardiologists.

Other risk factors: dyslipidaemia was defined as fasting serum TC levels $>5.6 \mathrm{mmol} / \mathrm{l}$, fasting serum TG levels $>1.7 \mathrm{mmol} / \mathrm{l}$, and fasting serum LDL levels $>3.4 \mathrm{mmol} / \mathrm{l}$ [20]. In addition, subjects who were previously diagnosed and who were taking lipid-lowering drugs.

Smoking [33]: Smoking was defined according to the standards formulated by the World Health Organization as continuous or accumulated smoking for 6 months or more than at least one cigarette per day.

Drinking [24]: Drinking was defined as the consumption of alcohol at least once per month, including $120 \mathrm{ml}$ of wine, $360 \mathrm{ml}$ of beer, or $45 \mathrm{ml}$ of spirits.

Additionally, a family history of high-risk diseases including stroke, coronary atherosclerotic heart disease, diabetes mellitus, hypertension, and hyperlipidaemia were also recorded. The above-mentioned related factors were classified according to their presence or absence.

\section{Statistical analysis}

All data were analysed using SPSS 17.0 software (IBM, USA). For multiple comparisons, each value was compared by one way ANOVA following Dunnett test when each datum conformed to normal distribution, while the non-normally distributed continuous data were compared using non-parametric tests. Logistic regression analysis was used for the multivariate analysis. Variables with a $p<0.1$ in the univariate comparisons were entered into multivariate stepwise logistic regression analysis. The 95\% confidence intervals $(\mathrm{Cl})$ and $p$ values were calculated. A value of $p<0.05$ (two-sided) was considered statistically significant. The statistical procedures were repeated for the discrimination of LAA and the control group, as well as LAA and cerebral SVD group.

\section{Results \\ Demographic data of the studied patient population}

There were 618 hospitalized patients with firstever ischemic stroke, we excluded 96 patients with cardioembolism, 114 patients with stroke of other undetermined aetiology, 39 patients with acute stroke of other determined aetiology, 4 patients with renal insufficiency, 6 patients with mixed stroke, 8 patients with tumour disease and 16 patients with other diseases. There were 223 male and 130 female patients aged from 35 to 89 years. Based on their clinical symptoms, signs, and imaging results, all of the patients were divided into an ischemic cerebral SVD group and an LAA group according to the TOAST criteria. The LAA group $(n=171)$ contained 97 men and 74 women ( $61.0 \pm 10.1$ years). The ischemic cerebral SVD group $(n=182)$ comprised 126 men and 56 women $(62.3 \pm 9.8$ years). A total of 70 cranial MRI-confirmed non-stroke patients older than 35 years who were admitted to the hospital during the same period served as the control group (42 men and 28 women, $54.6 \pm 8.9$ years). Controls had no history of stroke, and were matched with cases for age and sex. These patients had migraine, hypokalemic periodic paralysis, idiopathic facial paralysis, hypertension, or trigeminal neuralgia. Control participants did not have bleeding tendencies, cerebral aneurysms, arteriovenous malformations, mental illness, severe liver, kidney, heart, or lung diseases, autoimmune diseases, or cerebral SVD. There was no significant difference in the general information between the two groups $(p>0.05)$. A flow chart of the study selection process is shown.

\section{Risk factors for cerebral SVD}

The comparisons of risk factors between cerebral SVD and the control group were shown in Table I, in order to select the variables into the subsequent logistic regression. The multivariate logistic regression showed that hypertension $(\mathrm{OR}=6.706,95 \% \mathrm{Cl}=3.431$ 13.106, $p<0.001)$, diabetes mellitus ( $\mathrm{OR}=4.023,95 \%$ $\mathrm{Cl}=1.635-9.898, p=0.002)$, high TC levels $(\mathrm{OR}=5.327$, $95 \% \mathrm{Cl}=1.450-19.579, p=0.012)$, high TG levels (OR = $2.519,95 \% \mathrm{Cl}=1.174-5.405, p=0.018)$ and smoking 
$(\mathrm{OR}=2.998,95 \% \mathrm{Cl}=1.293-6.954)$ were significantly associated with ischemic cerebral SVD (Table I).

\section{Risk factors for LAA}

The comparisons ofriskfactors between LAAand the control group were shown in Table II. Multivariate logistic regression showed that hypertension $(O R=9.316$, $95 \% \mathrm{Cl}=4.503-19.273, p<0.001)$, diabetes mellitus $(\mathrm{OR}=3.866,95 \% \mathrm{Cl}=1.556-9.908, p=0.004)$, high $\operatorname{LDL}(\mathrm{OR}=2.620,95 \% \mathrm{Cl}=0.958-7.163, p=0.030)$, high TG $(\mathrm{OR}=2.601,95 \% \mathrm{Cl}=1.207-5.606, p=0.015)$ and smoking $(\mathrm{OR}=3.717,95 \% \mathrm{Cl}=1.555-8.882$, $p=0.003)$ were associated with LAA (Table II).

\section{Variables discriminating cerebral SVD and LAA}

In this analysis, the dependent variables were divided into cerebral SVD or LAA. Multivariate logistic regression showed that male sex $(O R=1.961$, $95 \% \mathrm{Cl}=1.160-3.315, p=0.012)$ and high TC lev-

Table I. Multivariate logistic regression (MLR) analysis results of risk factors of the ischemic cerebral SVD group and the control group

\begin{tabular}{|lcccc|}
\hline Related factors & $\beta$ & $P$ value & OR & $95 \% \mathrm{Cl}$ \\
\hline Hypertension & 1.903 & $<0.001$ & 6.706 & $3.431-13.106$ \\
\hline Diabetes mellitus & 1.392 & 0.002 & 4.023 & $1.635-9.898$ \\
\hline High TC & 1.673 & 0.012 & 5.327 & $1.450-19.579$ \\
\hline High TG & 0.924 & 0.018 & 2.519 & $1.174-5.405$ \\
\hline Smoking & 1.098 & 0.011 & 2.998 & $1.293-6.954$ \\
\hline
\end{tabular}

SVD - cerebral small vessel disease, TC - total cholesterol, TG - triglyceride

Table II. Multivariate logistic regression (MLR) analysis results of risk factors of the LAA group and the control group

\begin{tabular}{|lcccc|}
\hline Related factors & $\beta$ & $P$ value & OR & $95 \% \mathrm{Cl}$ \\
\hline Hypertension & 2.232 & $<0.001$ & 9.316 & $4.503-19.273$ \\
\hline Diabetes mellitus & 1.352 & 0.004 & 3.866 & $1.556-9.608$ \\
\hline High LDL & 0.963 & 0.030 & 2.620 & $0.958-7.163$ \\
\hline High TG & 0.956 & 0.015 & 2.601 & $1.207-5.606$ \\
\hline Smoking & 1.313 & 0.003 & 3.717 & $1.555-8.882$ \\
\hline
\end{tabular}

$L A A$ - large artery atherosclerosis, $L D L$ - low density lipoprotein, $T G$ - triglyceride

Table III. The comparison of the risk factors among the SVD group, LAA group and control group

\begin{tabular}{|c|c|c|c|}
\hline Variables & $\begin{array}{c}\text { Control } \\
n=70 \\
n(\%)\end{array}$ & $\begin{array}{c}\text { SVD } \\
n=182 \\
n(\%)\end{array}$ & $\begin{array}{c}\text { LAA } \\
n=171 \\
n(\%)\end{array}$ \\
\hline Age (year) & $54.6 \pm 8.9$ & $62.3 \pm 9.8$ & $61.0 \pm 10.1$ \\
\hline Male & $42(60.0)$ & $126(69.2)$ & $97(56.7)^{c \star}$ \\
\hline Hypertension & $20(28.5)$ & $131(71.9)^{a \S}$ & $125(73.1)^{\mathrm{b} \S}$ \\
\hline Diabetes mellitus & $9(12.8)$ & $57(31.3)^{\mathrm{a}^{\star \star}}$ & $53(31.0) b^{\star *}$ \\
\hline CAD & $6(8.5)$ & $21(11.5)$ & $28(16.4)$ \\
\hline Family history & $29(41.4)$ & $53(29.1)$ & $64(37.4)$ \\
\hline High TC & $4(5.7)$ & $17(9.3)^{\mathrm{a} \S}$ & $6(17.5)^{b * \star c \star *}$ \\
\hline High TG & $15(21.4)$ & $71(39.0)^{a t}$ & $76(44.4)^{\mathrm{bt}}$ \\
\hline High LDL & $8(11.4)$ & $48(26.3)^{a * \star}$ & $40(23.4)^{b * *}$ \\
\hline Smoking & $13(18.5)$ & $57(31.3)^{a \star \star}$ & $57(35.3)^{b * \star}$ \\
\hline Drinking & $17(24.2)$ & 40 (21.9) & 32 (18.7) \\
\hline
\end{tabular}

a SVD vs. control; ${ }^{b}$ LAA VS. control; ${ }^{c} S V D$ VS. LAA

${ }^{*} 0.1<p<0.05 ;{ }^{* *} p<0.05 ;{ }^{\dagger} p<0.01 ;{ }^{\S} p<0.001$

$S V D$ - cerebral small vessel disease, TG - triglyceride, $L D L$ - low-density lipoprotein, TC - total cholesterol, LAA - large artery atherosclerosis 
Table IV. Multivariate logistic regression (MLR) analysis results of risk factors of the ischemic cerebral SVD group and LAA group

\begin{tabular}{|lcccc|}
\hline Related factors & $\beta$ & $P$ value & OR & $95 \% \mathrm{Cl}$ \\
\hline High TC & 0.636 & 0.016 & 1.888 & $1.123-3.174$ \\
\hline Male & 0.481 & 0.012 & 1.961 & $1.160-3.315$ \\
\hline
\end{tabular}

SVD - cerebral small vessel disease, LAA - large artery atherosclerosis, TC - total cholesterol

els $(\mathrm{OR}=1.888,95 \% \mathrm{Cl}=1.123-3.174, p=0.016)$

were more likely to be associated with cerebral SVD (Tables III and IV).

\section{Discussion}

The present study analysed the risk factors and differences for ischemic cerebral SVD and LAA stroke using the TOAST criteria in patients who were treated at a tertiary hospital in Xingtai and yielded the following results. Firstly, hypertension, diabetes mellitus, high TC, hypertriglyceridemia and smoking were found to be strongly associated with ischemic cerebral SVD, whereas hypertension, diabetes mellitus, high LDL, hypertriglyceridemia and smoking were associated with LAA. Secondly, the result of the multivariate logistic regression analysis demonstrated that among the risk factors for ischemic cerebral SVD compared with those of, high TC was prominent risk factors for ischemic cerebral SVD.

Atherosclerosis is a lifelong disease that progresses over time, but the precise aetiology and risk factors for atherosclerosis are unclear. About 30\% of ischemic strokes is caused by large artery atherosclerosis (LAA), which is called LAA type stroke. It is an important cause of disability and death in China [11]. However, certain abnormal physiological conditions, traits, or habits may promote the development of atherosclerosis. The most common risk factors include high cholesterol and LDL levels, low high-density lipoprotein ( $\mathrm{HDL}$ ) levels, hypertension, smoking tobacco, diabetes mellitus, obesity, inactive lifestyle and advanced age.

Hypertension and diabetes were the major risk factors identified in LAA. In the early stage, oxidative stress damages the biological activity of nitric oxide produced by vascular endothelial cells, damages vascular endothelial cells, and causes reduced vascular compliance, that is, changes in vascular function. If the changes in vascular function cannot be detected in time and necessary interventions are carried out, structural changes will inevitably occur as the disease progresses further. Once developed to the stage of structural changes, the changes in arterial elasticity cannot be reversed [7].

Smoking is an independent risk factor for ischemic stroke [18]. In our study, we found that smoking is closely related to both atherosclerosis and ischemic cerebral SVD. The mechanisms through which smoking affects stroke risk are not fully clear, smoking is likely to contribute to an increased stroke risk through both short-term effect on thrombus generation in atherosclerotic arteries and long-term effect related to the development of atherosclerotic stenosis [4]. Smoking could cause stroke also through reversible factors such as increased platelet aggregation and arterial vasoconstriction by augmenting sympathetic activity, explaining the stroke risk [29]. A 10-year prospective cohort study in China found that smoking could raise the risk of ischemic stroke among hypertensives. Significant interaction was detected between smoking and hypertension on the risk of ischemic stroke [10].

In recent years, several epidemiologic studies have shown that dyslipidaemia is closely linked to the development and occurrence of ischemic stroke [13]. Lipid metabolism would be involved not only in large vessel atherosclerosis but also in the occlusive disease of small intra-cerebral arteries. Dyslipidaemia may be a causal and modifiable risk factor for ischemic stroke [36]. However, few studies in the literature have reported the relationship between stroke subtypes and lipid profiles. We also studied the different associations between two important subtypes of ischemic stroke and lipid profiles. We found that hypertriglyceridemia is closely associated with ischemic cerebrovascular disease and atherosclerotic cerebral infarction. High LDL is closely related to LAA, while hypercholesterolemia is more closely related to ischemic SVD.

Our study found that hypertriglyceridemia is related to ischemic stroke. Laloux et al. [12] found that hypertriglyceridemia was commonly detected in patients with various stroke subtypes, including cerebral SVD, which is similar to the result of the present study. It is demonstrated that hypertriglycer- 
idemia may play a role in atherosclerosis not only of large cerebral vessels but also of small penetrating arteries. Few studies outside of China have reported that hypertriglyceridemia plays a role in the development of ischemic cerebral SVD. The reason may be that Chinese and European people have different dietary structures, eating habits and sports activities. A previous study [30] revealed that serum TC and TG levels were increased in individuals who smoke and that alcohol consumption increased fasting TG levels, which ultimately led to dyslipidaemia and high TG levels. High TG levels are considered to be an independent risk factor for ischemic cerebral SVD in Chinese populations, which needs to be verified through further prospective multicentre studies. But one study showed that triglyceride lowering may not yield benefits in ischemic stroke and its subtypes [8].

Studies showed that LDL cholesterol (LDL-C) and TC levels may be independent predictors for the occurrence of large-artery atherosclerotic stroke $[8,12]$. The atherogenesis induced by LDL-C deposition in large vessels, and clinical studies have shown that an elevated blood LDL-C level is significantly related to the development of atherosclerotic lesions in large vessels [6]. However, in this study, high LDL was closely related to LAA, while hypercholesterolemia was closely related to ischemic cerebral SVD.

According to our results, it is speculated that hypercholesterolemia may also be involved in the aetiology of SVD [12]. By raising plasma cholesterol levels in Ldlr-/- mice fed a high-cholesterol diet, Kraft verified the hypothesis that hypercholesterolemia leads to pathological changes in small blood vessel walls and thrombosis, indicating that hypercholesterolemia plays an important role in the initiation of microvascular injury. Hypercholesterolemia causes upregulation of endothelial proteins (such as p-selectin), increased blood viscosity, increased shear sensitivity of neutrophils in microvascular beds, and changes in self-regulation leading to microvascular inflammatory thrombosis [17].

In our study, the comparative results of the risk factors for ischemic cerebral SVD and LAA demonstrated that high TC levels were strongly associated with ischemic cerebral SVD. In most studies, the incidence of lacunar infarction is higher in males [1]. Firstly, men are more likely than women to have unhealthy living habits, such as smoking and alcoholism, which are associated with cerebral arterioscle- rosis, hyaline arterioles, and even ischemic cerebral SVD [28]. With the same level of plasma cholesterol, men have twice the risk of cerebrovascular disease than women [14]. The literature has reported that Asian people are more likely to develop hypertension at an earlier age than people of other races. It has been suggested that patients from Asian populations with a longer history of hypertension may suffer more serious damage to small vessels in the brain [16]. Men lack the protection of endogenous stimulating hormones, and the rates of awareness, treatment and control of hypertension are low in males [21]. Secondly, You et al. found that plasma TC concentrations were higher in patients with small arterial stroke than in those with other subtypes of TOAST scores [35]. When serum TC concentrations are increased, cholesterol accumulates in the tunica intima of the arteries, especially in perforating branches, which may lead to atherosclerotic plaques and arteriolosclerosis.

Ischemic cerebral SVD and LAA have some common risk factors and some different risk factors. Therefore, measures for addressing the risk factors for ischemic cerebral SVD may differ from those for addressing the risk factors for LAA. The pathogenesis and classification of and targeted therapies for ischemic cerebral SVD need to be examined further.

The limitations of this study should also be considered. First, as a single-centre trial, the number of patients in the control group was small. Another study with a large sample size is still needed in the future. Second, the retrospective design cannot determine the causality of the associations. Third, in this study, we did not divide participants according to age which should be further researched in the future.

\section{Funding}

This work was supported by the Hebei Provincial Science Plan Project in China (13277757D).

\section{Ethics approval and consent to participate}

This study was conducted in accordance with the Declaration of Helsinki and approved by the Ethics Committee of Xingtai People's Hospital. Written informed consent has been obtained from all participants.

\section{Disclosure}

The authors report no conflict of interest. 


\section{References}

1. Arboix A, Martí-Vilalta JL. Lacunar stroke. Expert Rev Neurother 2009; 9: 179-196.

2. Blanco PJ, Müller LO, Spence JD. Blood pressure gradients in cerebral arteries: a clue to pathogenesis of cerebral small vessel disease. Stroke Vasc Neurol 2017; 2: 108-117.

3. Bullmore E, Sporns O. The economy of brain network organization. Nat Rev Neurosci 2012; 13: 336-349.

4. Burns DM. Epidemiology of smoking-induced cardiovasculardisease. Prog Cardiovasc Dis 2003; 46: 11-29.

5. Chinese Diabetes Society. Guidelines for the prevention and treatment of type 2 diabetes in China. Peking University Press, Beijing 2011; 5-6.

6. Deng QW, Liu YK, Zhang YQ, Chen XL, Jiang T, Hou JK, Shi HC, Lu M, Zhou F, Wang W, Li S, Sun HL, Zhou JS. Low triglyceride to high-density lipoprotein cholesterol ratio predicts hemorrhagic transformation in large atherosclerotic infarction of acute ischemic stroke. Aging 2019; 11: 1589-1601.

7. Gao YY, Wang HP, Zhang J. Effect of rosuvastatin on atherosclerosis and large artery elasticity in patients with hypertension and type 2 diabetes. Henan Med Res 2014; 23: 99-101.

8. Hindy G, Engström G, Larsson SC, Traylor M, Markus HS, Melander O, Orho-Melander M, Stroke Genetics Network $(\mathrm{SiGN})$. Role of blood lipids in the development of ischemic stroke and its subtypes a Mendelian randomization study. Stroke 2008; 49: 820-827.

9. Hou D, Liu J, Feng R, Gao Y, Wang YJ, Wu JL. The role of high-sensitivity C-reactive protein levels in functional outcomes in patients with large-artery atherosclerosis and small artery occlusion. Neurol Res 2017; 39: 981-987.

10. Huang FX, Zhu Z, Zhong C, Bu XQ, Zhou YP, Tian YF, Batu B, XU T, Wang AL, Li HM, Zhang MZ, Zhang YH. Smoking, hypertension, and their combined effect on ischemic stroke incidence: a prospective study among inner Mongolians in China. J Stroke Cerebrovasc Dis 2017; 26: 2749-2754.

11. Kim D, Park JM, Kang K, Cho YJ, Hong KS, Lee KB, Park TH, Lee SJ, Kim JG, Han MK, Kim BJ, Lee J, Cha JK, Kim DH, Nah HW, Kim DE, Ryu WS, Kim JT, Choi KH, Choi JC, Lee BC, Yu KH, Oh MS, Kim WJ, Kwon JH, Shin DI, Sohn SI, Hong JH, Lee JS, Lee JY, Gorelick PB, Bae HJ. Dual versus mono antiplatelet therapy in large atherosclerotic stroke. Stroke 2019; 50: 1184-1192.

12. Laloux P, Galanti L, Jamart J. Lipids in ischemic stroke subtypes. Acta Neurol Belg 2004; 104: 13-19.

13. Lee JS, Chang PY, Zhang Y, Kizer JR, Best LG, Howard BV. Triglyceride and HDL-C dyslipidemia and risks of coronary heart disease and ischemic stroke by glycemic dysregulation status: The Strong Heart Study. Diabetes Care 2017; 4: 529-537.

14. Lindenstrøm E, Boysen G, Nyboe J. Influence of total cholester$\mathrm{ol}$, high density lipoprotein cholesterol, and triglycerides on risk of cerebrovascular disease: the Copenhagen city heart study. BMJ 1994; 309: 11-15.

15. Liu LS. Writing Group of 2010 Chinese Guidelines for the management of hypertension [2010 Chinese guidelines for the management of hypertension]. Zhonghua Xin Xue Guan Bing Za Zhi 2011; 39: 579-615.
16. Liu YY, Lv P, Jin HQ, Cui W, Niu CG, Zhao MM, Fan CH, Teng YM, Pan B, Peng Q, Luo JJ, Zheng LM, Huang YN. Association between low estimated glomerular filtration rate and risk of cerebral small-vessel diseases: a meta-analysis. J Stroke Cerebrovasc Dis 2016; 25: 710-716.

17. Lopes FG, Bottino DA, Oliveira FJ, Mecenas AS, Clapauch R, Bouskela E. In elderly women moderate hypercholesterolemia is associated to endothelial and microcirculatory impairments. Microvasc Res 2013; 85: 99-103.

18. MarkidanJ,ColeJW,CroninCA,MerinoJG, PhippsMS, WozniakMA, Kittner SJ. Smoking and risk of ischemic stroke in young men. Stroke 2018; 49: 1276-1278.

19. Mcardle PF, Kittner SJ, Ay H, Brown Jr RD, Meschia JF, Rundek T, Wassertheil-Smoller S, Woo D, Andsberg G, Biffi A, Brenner DA, Cole JW, Corriveau R, de Bakker PIW, Delavaran H, Dichgans M, Grewal RP, Gwinn K, Huq M, Jern C, Jimenez-Conde J, Jood K, Kaplan RC, Katschnig P, Katsnelson M, Labovitz DL, Lemmens R, Li LX, Lindgren A, Markus HS, Peddareddygari LR, Pedersén A, Pera J, Redfors P, Roquer J, Rosand J, Rost NS, Rothwell PM, Sacco RL, Sharma P, Slowik A, Sudlow C, Thijs V, Tiedt S, Valenti R, Worrall BB, NINDS SiGN Study. Agreement between TOAST and CCS ischemic stroke classification: the NINDS SiGN Study. Neurology 2014; 83: 1653-1660.

20. Medical Administration Department of the Ministry of Health in People's Republic of China. National Clinical Test Procedures. Southeast University Press, Nanjing 2006; 474-496.

21. Moon JY, Park KJ, Hwangbo Y, Lee MR, Yoo BI, Won JH, Park YH. A trend analysis of the prevalence, awareness, treatment, and control of hypertension by age group. J Prev Med Public Health 2013; 46: 353-359.

22. Nam KW, Kwon HM, Lim JS, Han MK, Nam H, Lee YS. The presence and severity of cerebral small vessel disease increases the frequency of stroke in a cohort of patients with large artery occlusive disease. PLoS One 2017; 12: e0184944.

23. Nam KW, Kwon HM, Lim JS, Han MK, Nam H, Lee YS. The presence and severity of cerebral small vessel disease increases the frequency of stroke in a cohort of patients with large artery occlusive disease. PLoS One 2017; 12: e0184944.

24. National Blood Pressure Sampling Survey Collaborative Group. Drinking and blood pressure: sampling results in 1991. Zhonghua Gao Xue Ya Za Zhi 1995; 3: 52-56.

25. Pantoni L. Cerebral small vessel disease: from pathogenesis and clinical characteristics to therapeutic challenges. Lancet Neurol 2010; 9: 689-701.

26. Romero JR, Preis SR, Beiser A, DeCarli C, D’Agostino RB, Wolf PA, Vasan RS, Polak JF, Seshadri S. Carotid atherosclerosis and cerebral microbleeds: the Framingham heart study. J Am Heart Assoc 2016; 5: e002377.

27. Shi Y, Wardlaw JM. Update on cerebral small vessel disease: a dynamic whole-brain disease. Stroke Vasc Neurol 2016; 1: 83-92.

28. Tanaka A, Cui R, Kitamura A, Liu KY, Imano H, Yamagishi K, Kiyama M, Okada T, Iso H, CIRCS Investigators. Heavy alcohol consumption is associated with impaired endothelial function. J Atheroscler Thromb 2016; 23: 1047-1054.

29. Torrealba-Acosta G, Carazo-Céspedes K, Chiou SH, O’Brien AT, Fernández-Morales H. Epidemiology of stroke in Costa Rica: 
A 7-year hospital-based acute stroke registry of 1319 consecutive patients. J Stroke Cerebrovasc Dis 2018; 27: 1143-1152.

30. Wang T. Epidemiological characteristics and influencing factors of dyslipidemia in adults. Zhiye Jiankang 2015; 7: 1000-1004.

31. Wardlaw JM, Smith C, Dichgans M. Mechanisms of sporadic cerebral small vessel disease: insights from neuroimaging. Lancet Neurol 2013; 12: 483-497.

32. Wardlaw JM, Smith EE, Biessels GJ, Cordonnier C, Fazekas F, Frayne R, Lindley RI, O'Brien JT, BarkhofF, Benavente OR, Black SE, Brayne C, Breteler M, Chabriat H, Decarli C, de Leeuw F, Doubal F, Duering M, Fox NC, Greenberg S, Hachinski V, Kilimann I, Mok V Oostenbrugge RV, Pantoni L, Speck O, Stephan BCM, Teipel S, Viswanathan A, Werring D, Chen C, Smith C, Buchem MV, Norrving B, Gorelick PB, Dichgans M, STandards for ReportIng Vascular changes on nEuroimaging (STRIVE v1). Neuroimaging standards for research into small vessel disease and its contribution to ageing and neurodegeneration. Lancet Neurol 2013; 12: 822-838.

33. World Health Organization. Guidelines for controlling and monitoring the tobacco epidemic. World Health Organization, Geneva 1997; 190.

34. Wu CY, Wu HM, Lee JD, Weng HH. Stroke risk factors and subtypes in different age groups: a hospitalbased study. Neurol India 2010; 58: 863-868.

35. You N, Liu X, Shi H, Shen XL, Chen X, Lu YJ, Ding Q. Analysis on risk factors for different TOAST subtypes of patients with acute cerebral infarction. Shen Jing Ji Bing and Jing Shen Wei Sheng 2009; 9: 342-343.

36. Yuan BB, Luo GG, Gao JX, Qiao J, Yang JB, Huo K, Li YB, Liu Y. Variance of serum lipid levels in stroke subtypes. Clin Lab 2015; 61: 1509-1514 